\title{
Immunogenicity of insulin-producing cells derived from human umbilical cord mesenchymal stem cells
}

\author{
XIAO-FEI YANG ${ }^{1-3^{*}}$, TAO CHEN ${ }^{1-3^{*}}$, LI-WEI REN $^{1-3}$, LU YANG $^{1-3}$, HUI QI ${ }^{1,3}$ and FU-RONG LI $^{1-3}$ \\ ${ }^{1}$ The Key Laboratory of Stem Cell and Cellular Therapy, The Second Clinical Medical College \\ (Shenzhen People's Hospital), Jinan University, Shenzhen, Guangdong 518020; \\ ${ }^{2}$ School of Medicine, Jinan University, Guangzhou, Guangdong 510632; \\ ${ }^{3}$ Shenzhen Cell Therapy Public Service Platform, Shenzhen, Guangdong 518020, P.R. China
}

Received September 12, 2016; Accepted December 28, 2016

DOI: $10.3892 / \mathrm{etm} .2017 .4096$

\begin{abstract}
Mesenchymal stem cells (MSCs) have been considered as hypo-immunogenic and immunosuppressive. However, a thorough understanding of the immunological properties after MSC differentiation in vitro and in vivo has not been reached. We asked whether it would be immunogenic after differentiation or influenced by the immune microenvironment after transplantation. In different disease models, the immunological changes of MSCs after differentiation greatly varied, with contradicting results. In order to clarify this, we used a modified four-step induction method to induce human umbilical cord MSCs (hUCMSCs) to differentiate into insulin-producing cells (IPCs), and investigate the immunological changes after differentiation and immune reactions after transplantation into diabetic mice. We found that the induced IPCs are hypo-immunogenic, lacking HLA-DR, CD40 and CD80 expression. Of note, we observed immune cell infiltration to peritoneal cavity and left kidney capsule after local transplantation of induced IPCs. This indicated that hUCMSC-derived IPCs maintained hypo-immunogenic in vitro, but became immunogenic after transplanting to the host, possibly due to the changes of immune microenvironment and thereafter immunological enhancement and immune cell infiltration.
\end{abstract}

\section{Introduction}

Islet transplantation has progressed to be a possible cure for type 1 diabetes mellitus (1), but is clinically limited due

Correspondence to: Dr Fu-Rong Li, The Key Laboratory of Stem Cell and Cellular Therapy, The Second Clinical Medical College (Shenzhen People's Hospital), Jinan University, 1017 Dongmen North Road, Shenzhen, Guangdong 518020, P.R. China

E-mail: frli62@163.com

*Contributed equally

Key words: human umbilical cord mesenchymal stem cells, differentiation, insulin-producing cells, immunogenicity, diabetes mellitus to the shortage of donor islets and immune rejection (2). Mesenchymal stem cells (MSCs) are multipotent stromal cells that can differentiate into a variety of cell types, and can be easily derived from many different tissues with no ethic and legal issues. Most importantly is that they have been reported to exhibit unique immunomodulation properties, which make them optimal cell types in treating autoimmune diseases and graft versus host disease (GvHD). MSCs have been successfully induced to differentiate into insulin-producing cells (IPCs) in vitro, and these IPCs may be a new cell source for islet transplantation, thus they have great potential in cell replacement therapy for diabetes (3-5).

MSCs express low levels of class I major histocompatibility complex (MHC) molecules, but not class II and co-stimulatory molecules. MSCs suppress lymphocyte proliferation in vitro when co-cultured with PBMCs, and exert resistance against the cytotoxicity of cytotoxic lymphocytes. Due to their hypo-immunogenicity and immunosuppressive properties (6-8), clinical trials on allogenic MSCs injection in many different acute and chronic diseases have been registered and progressed (https://clinicaltrials.gov). However, MSCs may become immunogenic after differentiation and transplantation to host, due to induction process and the microenvironment of transplanted sites (9-14). In vitro differentiation of rat bone marrow-derived MSCs into muscle cells caused elevated expression of MHC-Ia and MHC-II, and became immunogenic. After transplantation to the infracted myocardium of allogenic rat, their survival and repair effects were much weaker than those of autologous transplantation (12). The induction process of muscle cells in vitro could reduce the secretion of immunomodulatory molecule PEG2, thus influence the survival of the differentiated cells in the host (15). The situation was similar when bone marrow-derived MSCs were induced into chrondocytes in vitro. The differentiated chondrocytes with increased immunogenicity could promote DC maturation, stimulate PBMC proliferation and activate cytotoxic $\mathrm{T}$ lymphocytes (14). We were interested in the immunogenic changes after MSC differentiation into IPCs in vitro and, after transplantation into the diabetic model. Therefore, we induced human umbilical cord MSCs (hUCMSCs) to differentiate into IPCs in vitro and transplanted these differentiated cells into diabetic mice to determine whether they could combat against 
hyperglycemia. We investigated the immunological properties of the differentiated IPCs in vitro, including the expression of HLA-ABC, HLA-DR, the costimulatory molecules CD40 and CD80, and stimulation of allogenic PBMCs proliferation. On immunogenic changes of the IPCs after transplantation into diabetic mice, we observed the immune cell infiltration of the transplanted sites.

\section{Materials and methods}

Isolation and characterization of hUCMSCs. Institutional Review Board approval was obtained for all the procedures. Following the written informed consent of parents, fresh human umbilical cords were obtained after birth from the Second Affiliated Hospital of Jinan University. hUCMSCs were isolated and cultured as previously described (16). The cells were cultured in Dulbecco's modified Eagle's medium (DMEM)/F12 (Gibco, Grand Island, NY, USA) with 10\% fetal bovine serum (FBS), $100 \mathrm{U} / \mathrm{ml}$ penicillin $\mathrm{G}$ and $100 \mathrm{mg} / \mathrm{ml}$ streptomycin and maintained for 3-5 days in a humidified incubator at $37^{\circ} \mathrm{C}$ with $5 \% \mathrm{CO}_{2}$. Flow cytometry on an Altra (Beckman Coulter, Fullerton, CA, USA) was performed to analyze characteristic MSC markers including CD29, CD34, CD90F, CD45, CD106 and HLA-DR (eBioscience, Inc., San Diego, CA, USA). hUCMSCs were also characterized by differentiation toward adipogenic and osteogenic lineages using previously described protocols (Alizarin red and Oil Red O assay) by commercial kits (Cyagen Biosciences Inc., Santa Clara, CA, USA).

Pancreatic $\beta$-cell differentiation. For pancreatic $\beta$-cell differentiation, hUCMSCs were induced with a modified four-step protocol with the addition of islet neogenesis-associated protein pentadecapeptide (INGAP-PP), as previously described $(17,18)$. Differentiation was induced by treating the cells with CMRL medium supplemented with $10 \%$ FBS (Gibco), 10 mM nicotinamide (Sigma-Aldrich, St. Louis, MO, USA), and $4 \mathrm{nM}$ activin A (R\&D Systems, Inc., Minneapolis, MN, USA), $25 \mathrm{ng} / \mathrm{ml}$ recombinate EGF (Invitrogen-Biosource, Carslbad, CA, USA), and $0.5 \mathrm{mM} \beta$-mercaptoethanol (Gibco) for 3 days. Then the cells were treated with second stage induction medium for 5 days. The second stage medium was CMRL medium supplemented with $10 \%$ FBS, 10 mM nicotinamide, $4 \mathrm{nM}$ activin $\mathrm{A}$, and $25 \mathrm{ng} / \mathrm{ml}$ recombinate EGF. At the third induction stage, the cells were cultured in DMEM/F12 medium supplemented with $2 \% \mathrm{FBS}, 10 \mathrm{mM}$ nicotinamide $10 \mathrm{nM}$ Exendin-4 (AnaSpec, Fremont, CA, USA), $10 \mu \mathrm{g} / \mathrm{ml}$ INGAP-pp (Sangon Biotech Co., Ltd., Shanghai, China) and 1X ITS (Gibco) for 7 days. An last induction stage, the cells were treated with high-glucose DMEM supplemented with $10 \mathrm{mM}$ nicotinamide, $10 \mathrm{nM}$ Exendin-4, $10 \mu \mathrm{g} / \mathrm{ml}$ INGAP-pp and 1XITS, and recombinant bFGF (Prospec, East Brunswick, NJ, USA) for 7 days.

RT-qPCR analysis of pancreatic developmental-related genes. hUCMSCs, day 14 IPCs, day 21 IPCs and human islets were harvested for gene expression analysis. The total RNA was isolated using TRIzol reagent (Takara Bio, Inc., Otsu, Japan), and was reverse-transcribed into cDNA using a PrimeScript RT Reagent kit with gDNA eraser (Takara) according to the manufacturer's instructions. Quantitative gene expression of Pdx1, Ngn3, MafA, NeuroD1, and insulin was determined using SYBR-Green Premix Ex Taq (Takara) and performed using standard methods as previously described (19). The primer sets used were: Pdx-1 forward, 5'-ttgaacttgaccgagagacaca-3' and reverse, 5'-cgcttcttgtcetcctcc ttt-3'; Ngn3 forward, 5'-cttcgccacaactacatctg-3' and reverse, 5'-ctgggagactggggagtagag-3'; NeuroD1 forward, 5'-tgaaagccetctgactgattg-3' and reverse, 5'-cggtgcetgagaagattgat-3'; MafA forward, 5'-cttcagcaaggaggaggtcat-3' and reverse, 5'-agttggcacttctcgctctc-3'; insulin forward, 5'-cagcctttgtgaaccaacac-3' and reverse, 5'-cgggtcttgggtgtgtagaa-3'; $\beta$-actin forward, 5'-ctgggac gacatggagaaaa-3' and reverse, 5'-aaggaaggctggaagagtgc-3'.

Immunofluorescence staining for pancreatic developmetal-related markers. hUCMSCs and day 21 IPCs were fixed in $4 \%$ paraformaldehyde for $20 \mathrm{~min}$ and blocked with $0.2 \%$ Triton X-100 supplemented with 2\% BSA for $1 \mathrm{~h}$. Cells were then incubated with a rabbit anti-human Pdx1 polyclonal antibody (1:100; cat. no. sc-25403) and MafA polyclonal antibody (1:100; cat. no. sc-66958) (both from Santa Cruz Biotechnology, Inc., Santa Cruz, CA, USA), rabbit anti-human C-peptide polyclonal antibody (1:100; cat. no. ab14181; Abcam, Cambridge, UK), goat anti-human Nkx6-1 polyclonal antibody (1:200; cat. no. MBS622560; Invitrogen-Biosource) at $4^{\circ} \mathrm{C}$ overnight. Then, cells were incubated with Rhodamine Red-conjugated goat anti-rabbit IgG Rhodamine Red antibody (1:200; cat. no. MBS235303; Invitrogen-Biosource) FITC-conjugated goat anti-rabbit IgG (1:1,000; cat. no. sc-2012) and FITC-conjugated donkey anti-goat IgG antibody (1:200; sc-2024) (both from Santa Cruz Biotechnology, Inc.). The nuclei were counterstained with DAPI (1:800), and cells were visualized under a fluorescence microscope (Eclipse TE2000-U; Nikon GmbH, Düsseldorf, Germany).

Immune antigen expression analysis. The immuno-phenotype (HLA-ABC, HLA-DR, CD40 and CD80) of hUCMSCs and day 21 IPCs was examined by flow cytometry on a flow cytometer (Epics Altra; Beckman Coulter) with specific phycoerythrin or FITC-conjugated monoclonal antibodies (eBioscience, Inc.) and performed as previously described (20). CellQuest software (BD Biosciences, Franklin Lakes, NJ, USA) was used for data analysis. Results are expressed as a percentage of positive cells or as mean relative fluorescence intensity, obtained as a ratio between the mean fluorescence intensity of cells stained with specific $\mathrm{mAb}$ and the mean fluorescence intensity obtained with isotype control.

Purification of IPCs. Newport Green (NG) is a fluorescent dye specifically binding to zinc. Insulin is stored in $\beta$ cells as crystals containing zinc, so NG can be used to label functional $\beta$ cells and NG diacetate (NG-AC) is suitable for live cell staining (21). Differentiated IPCs were washed twice with phosphate-buffered saline (PBS), then incubated with $1-10 \mu \mathrm{M}$ $\mathrm{NG}-\mathrm{AC}$ and $1 \mu \mathrm{l} / \mathrm{ml}$ Pluronic $\mathrm{F} 127$ at $37^{\circ} \mathrm{C}$ for $30 \mathrm{~min}$. After washing in PBS with 5\% FBS, the cells were re-suspended and was subjected to FACS analysis.

One-way mixed lymphocyte reaction. hPBMCs as effector cells were isolated from healthy donors following informed 
consent. hUCMSCs at passage 3 or day 21 IPCs pretreated with $25 \mathrm{mg} / \mathrm{ml}$ mitomycin C (Roche Diagnostics, Indianapolis, IN, USA) were used as stimulator cells. A total of $1 \times 10^{5}$ effector cells were co-cultured with $1 \times 10^{4}$ stimulator cells in 96-well plates. Effector cells treated with PHA $(5 \mathrm{mg} / \mathrm{ml}$; Sigma-Aldrich) were used as a positive control, and effector cells alone were used as a negative control. After co-culture for $72 \mathrm{~h}$, the proliferation of effector cells was assayed with a cell counting kit (Dojindo Laboratories, Kumamoto, Japan) and the $\mathrm{OD}$ at $450 \mathrm{~nm}$ was measured with a Bio-Rad 550 microplate reader (Bio-Rad, Tokyo, Japan). The SI was calculated using the formula: $\mathrm{SI}=$ (co-cultured sample OD - stimulator OD)/(negative control OD - blank OD).

Cell-mediated lympholysis (CML) test. C57BL/6 mice were randomly divided into two groups, hUCMSCs and IPCs group, 5 mice in each group. On day 1 and $6,1 \times 10^{7}$ hUCMSCs or purified IPCs were peritoneally injected to immune animals. On day 10, the mice were sacrificed. Spleen was removed and made to single cell suspension at a density of $1.0 \times 10^{7} / \mathrm{ml}$, as effector cells. A total of $1 \times 10^{5}$ cells of splenocytes was incubated with a serial titration of the same stimulating cells for $72 \mathrm{~h}$ at $37^{\circ} \mathrm{C}$. The cytotoxicity was evaluated by staining with Annexin V and PI, and the apoptosis rate was analyzed by FACS.

Interleukin (IL)-2, IL-4 and interferon (IFN)- $\gamma$ secretion in $C M L$. IL-2, IL-4 and IFN- $\gamma$ secretion was tested with the supernatants collected from the wells of CML plates. The assay procedure followed instructions of the mouse ELISA kit (4A Biotech Co., Ltd., Beijing, China). Absorbance was measured using a Bio-Rad 680 microplate reader (Bio-Rad) at $450 \mathrm{~nm}$.

For peritoneal inflammatory cell infiltration test male C57BL/6 mice with an initial body weight of 20-25 g were randomly assigned into three groups: hUCMSCs $(n=4), 1.0 \times 10^{7}$ hUCMSCs at passage 3 were injected into peritoneal cavity; IPCs $(\mathrm{n}=4), 1.0 \times 10^{7}$ purified IPCs were injected into peritoneal cavity; control group $(\mathrm{n}=4), 1 \mathrm{ml}$ PBS were intraperitoneally injected. Four-hour post-injection, peritoneal cavity lavage was performed by injecting $8 \mathrm{ml}$ PBS into the cavity. Then the lavage was centrifuged at $1,000 \mathrm{xg}$ for $5 \mathrm{~min}$ and treated with red blood cell lysis buffer at room temperature for $10 \mathrm{~min}$. The cells were then stained with FITC anti-mouse CD3e and PE anti-mouse CD45 and analyzed by FACS.

Cell transplantation and histological analysis. C57B/L mice were purchased from the Experimental Animal Center of Southern Medical University and housed under specific pathogen-free conditions. All animal procedures were approved by the Institutional Animal Care and Use Committee at Shenzhen PKU-HKUST Medical Center. Male mice with an initial body weight of 20-25 g were fasted for $12 \mathrm{~h}$ and then intraperitoneally injected with streptozotocin (Sigma-Aldrich) at a single dose of $170 \mathrm{mg} / \mathrm{kg}$. After $48 \mathrm{~h}$, tail vein blood samples were obtained for blood glucose (BG) measurements using a BG device (Roche Accu-Chek III; Roche Diagnostics, Basel, Switzerland). Mice with a non-fasting BG of $300 \mathrm{mg} / \mathrm{dl}$ for 3 consecutive days were considered diabetic. The diabetic animals were randomly assigned based on the transplanted
A
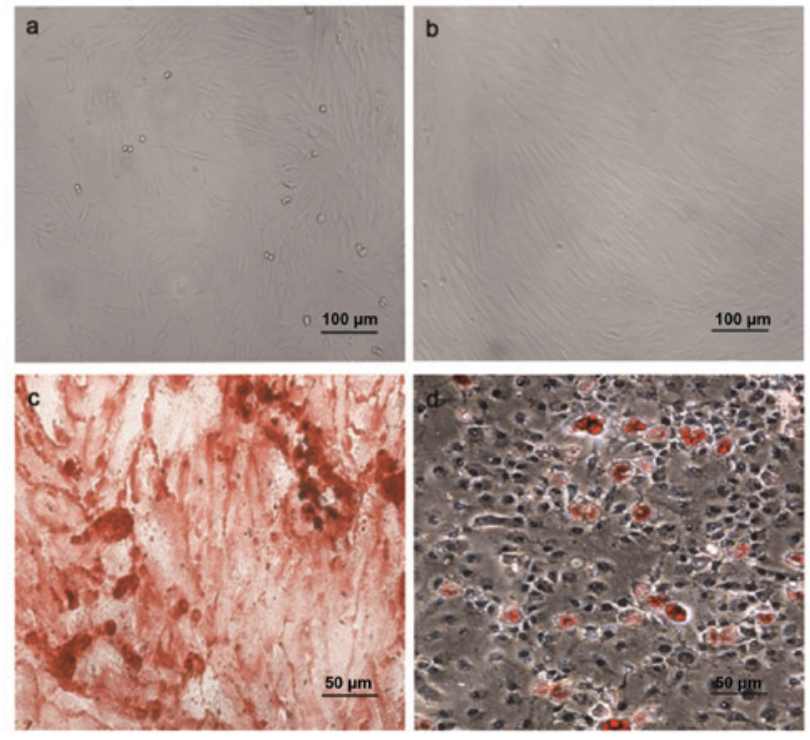

B
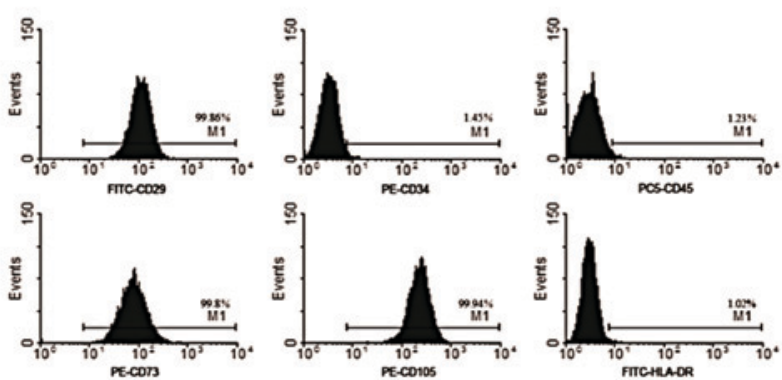

Figure 1. Characterization of hUCMSCs. (A) Morphology of hUCMSCs at (a) passage 1 and (b) 4: (c) hUCMSCs were induced to differentiate into osteogenic cells and stained positive for Alizarin red. (d) Under adipogenic conditions, hUCMSCs were positively stained by Oil Red $\mathrm{O}$ assay. (a and b) x100 and (c and d) x200 magnification. (B) Flow cytometric analysis of surface marker. hUCMSCs were positive for CD29, CD73, CD105, but negative for CD34, CD45 and HLA-DR. hUCMSCs, human umbilical cord mesenchymal stem cells.

cell types; $1.0 \times 10^{7}$ hUCMSCs or purified IPCs were transplanted under the left kidney capsule, marked as hUCMSCs $(n=10)$ and IPCs group $(n=10)$. Diabetic group $(n=10)$ and nondiabetic mice $(n=6)$ were injected with the same amount of PBS, as positive and negative control. Non-fasting BG levels were measured at a certain time every 3 days post-transplantation until day 30 , and the body weight was monitored at the same time.

The left kidney was removed from mice in hUCMSCs $(\mathrm{n}=3)$ and IPCs group $(n=3)$ on day 15 and 30 post-transplantation, and then fixed in $10 \%$ buffered formalin. Paraffin-embedded $5 \mu \mathrm{m}$ sections were routinely stained with hematoxylin and eosin. The hematoxylin and eosin-stained kidney sections were analyzed by counting the number of inflammatory cells infiltrating into the left kidney in 10 high-power fields ( $x 400$ ) per sample (22).

Statistical analysis. Data were expressed as the mean \pm standard deviation (SD). Statistical analysis was performed using one-way analysis of variance (ANOVA) to compare the difference among different groups. Independent sample t-tests were used to statistically compare the difference between 
A
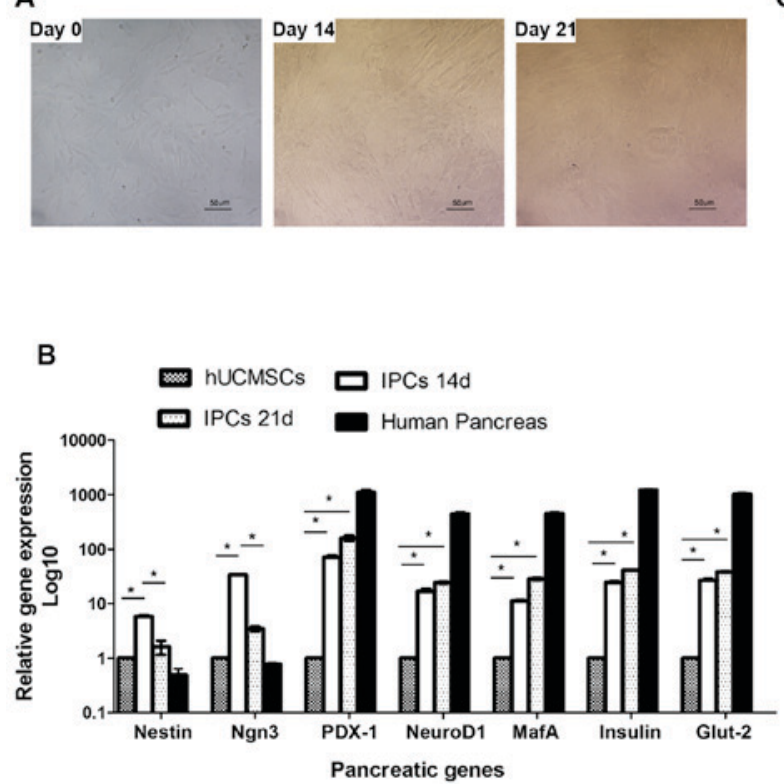

D
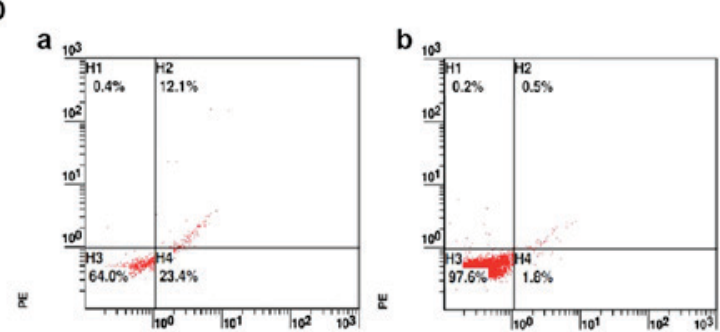

C
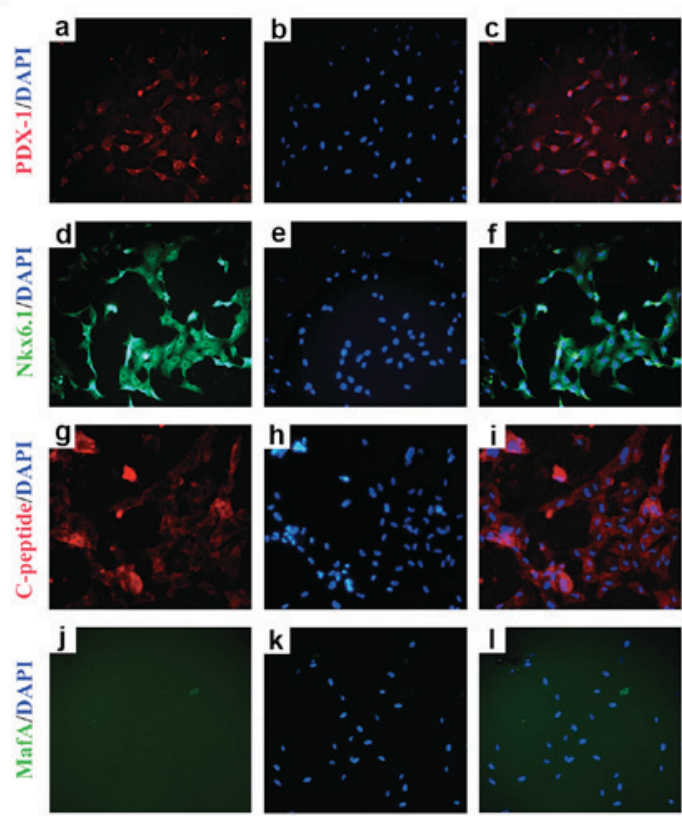

E

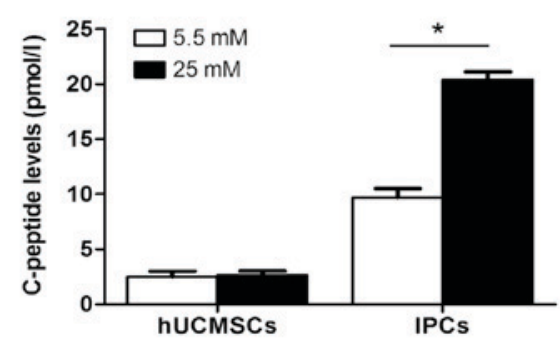

Figure 2. IPCs induction and function analysis. (A) Morphology changes during IPC induction from hUCMSCs on days 14 and 20 ; $x 200$ magnification. (B) mRNA levels of pancreatic developmental-related genes. Total mRNA was extracted to evaluate the expression of pancreatic genes (Nestin, Ngn3, Pdx1, NeuroD1, MafA, insulin and Glut-2) by RT-qPCR. * P<0.05. (C) Induced IPCs were stained positive for Pdx1, Nkx6.1 and C-peptide by immunofluorescence staining; x200 magnification. (D) Flow cytometric detection of c-peptide expression on induced IPCs. Induced IPCs on (a) day 21 and (b) isotype control. (E) Glucose challenge test, day 21 IPCs were incubated with low glucose $(5.5 \mathrm{mM})$ and high glucose $(25 \mathrm{mM})$ for $3 \mathrm{~h}$, then C-peptide release was measured in the supernatants. ${ }^{*} \mathrm{P}<0.01$. IPCs, insulin-producing cells; hUCMSCs, human umbilical cord mesenchymal stem cells.

two groups. Sequential data were compared using repeated measures ANOVA. $\mathrm{P}<0.05$ was considered to indicate a statistically significant difference.

\section{Results}

Isolation and characterization of hUCMSCs. After purification, hUCMSCs were spindle-shaped and attached to the plate during cell culture (Fig. 1A-a and b). Flow cytometric analysis demonstrated that these cells were positive for CD29 (99.86\%), CD73 (99.8\%), CD105 (99.94\%), but negative for CD45 (1.23\%), CD34 (1.45\%) and HLA-DR (1.02\%), which was in accordance with MSC characteristics as reported previously (Fig. 1B). After adipogenic induction, the cells were stained positive for Oil Red O, showing lipid-filled vesicles. After osteogenic induction, these cells displayed osteogenesis, as shown by Alizarin red staining of calcium deposits (Fig. 1A-c and d).

IPCs induction from hUCMSCs. Following the addition of INGAP-pp and certain small molecules, modified four-step induction protocol was used to induce IPCs from hUCMSCs. During induction, the cells started to become polygonal and rhombic on day 14 , then cells aggregated to grow, became oval and formed a regular pavement-like morphology on day 21 (Fig. 2A). The maturity of differentiated IPCs was investigated by immunofluorescent staining and glucose stimulation test. In immunofluorescent staining, differentiated IPCs expressed C-peptide, indicating the production of insulin, not absorption from the medium (Fig. 2C). Furthermore, these cells also expressed the mature $\beta$ cell markers PDX1, NKX6.1, but no MAFA (Fig. 2C), indicating moderate but not full maturity of the differentiated IPCs. Flow cytometric analysis showed that the rate of C-peptide expression of fully differentiated IPCs was $12.53 \pm 0.92 \%$, while almost no C-peptide positive cells were found in hUCMSCs (Fig. 2D). This indicated that about $12.03 \pm 0.92 \%$ cells were successfully differentiated into IPCs. The test of glucose stimulation showed that these induced IPCs secreted a low level of C-peptide (48.32 \pm 0.39$)$ with stimulation of low glucose (5.5 mM) (Fig. 2E). After incubation with high glucose $(25 \mathrm{mM}), \mathrm{C}$-peptide level in the supernatant were 3 -fold higher than that of low glucose (Fig. 2E). These results indicated that the induced IPCs by our modified protocol could secrete insulin in a glucose-regulated manner.

RT-qPCR analysis of the levels of pancreatic developmental gene $m R N A$. Induced IPCs were stained with NG and sorted 
A
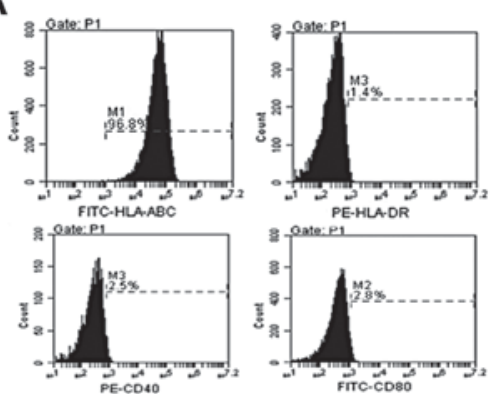

C

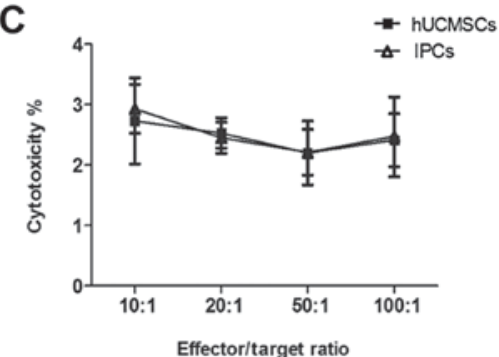

E

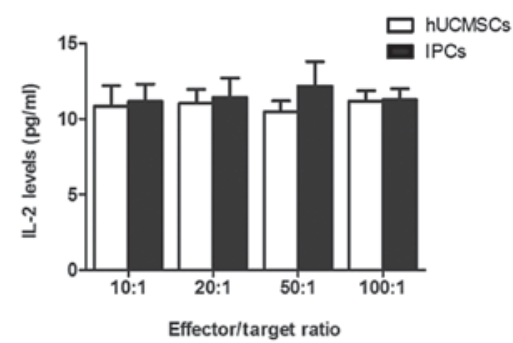

B
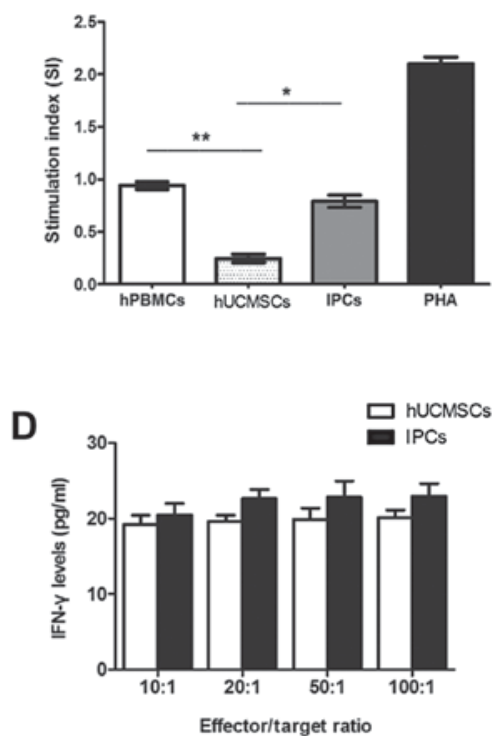

$\mathbf{F}$

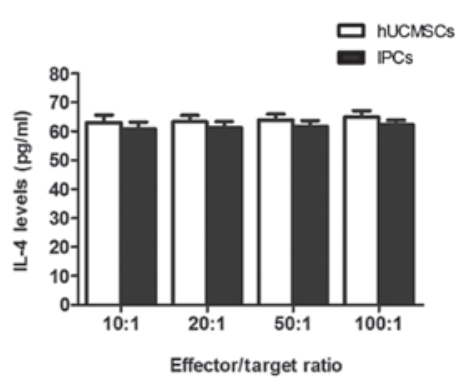

Figure 3. In vitro immunological characteristics of induced IPCs. (A) FACS shows that induced IPCs expressed MHC-I and did not express HLA-DR, CD40 and CD80. (B) Allogenic PBMCs were co-cultured with hUCMSCs or IPCs for $72 \mathrm{~h}$. No proliferation was observed in the IPCs group compared with the PBMCs auto-proliferation and PHA (positive) group. ${ }^{*} \mathrm{P}<0.05,{ }^{* *} \mathrm{P}<0.01$. (C) Splenocytes collected from recipients were considered as effector cells, and then co-cultured with IPCs (target cell to effector cell ratio: 1:10, 1:20, 1:50 and 1:100) for $72 \mathrm{~h}$. Percentage of apoptotic MSCs were evaluated by Annexin V-APC/PI staining and flow cytometry. (D-F) IL-2, IL-4 and IFN- $\gamma$ secretion in CML supernatants at different effector/target ratio. IPCs, insulin-producing cells; hUCMSCs, human umbilical cord mesenchymal stem cells; MSCs, mesenchymal stem cells; CML, cell-mediated lympholysis.

by FACS. Upon FACS analysis, 6-8\% of the cells were selected as positive for NG, no green fluorescence was observed in the control group (data not shown). RT-qPCR analysis was performed to investigate the changes of pancreatic developmental gene expression during induction and differentiation. In induced IPCs, Nestin, a marker for pancreatic development, was upregulated on day 14 and then decreased on day 21 ; the same trend was also observed on Ngn3-a pancreatic endocrine progenitor marker. Compared to hUCMSCs, Ngn3 mRNA was significantly upregulated $(\mathrm{P}<0.05)$ on day 14 , and its level was reduced on day 21. There was an increased expression of $\beta$-cell transcripts such as insulin, Pdx1, MafA, NeuroD1 and Glut2 on days 14 and 21, but albeit much lower than the level of adult human islets (Fig. 2B).

Immune antigen expression on IPCs and lymphocyte proliferation assay in vitro. FACS showed that the IPCs expressed high levels of HLA-ABC $(97.93 \pm 1.71)$, and no HLA-DR, CD40 and CD80 in vitro, which is similar to hUCMSCs without induction (Fig. 3A). In one-way mixed lymphocyte reaction assay, the stimulation index of hPBMSCs after co-culturing with hUCMSCs was $0.24 \pm 0.04$, which was significantly different compared with the positive control group (PHA) and negative control group (auto-hPBMCs) $(\mathrm{P}<0.05)$. The index of stimulation of IPC co-culture group was $0.79 \pm 0.06$, which was lower compared with the positive control group, while no difference was found with the negative control group $(\mathrm{P}>0.05)$ (Fig. 3B), indicating that induced IPCs had low immunogenicity in vitro, but did not have the immunosuppressive property as hUCMSCs did.

Cell-mediated lysis test. In order to observe sensitization of the host lymphocytes by the induced IPCs, we pre-sensitized the mice with hUCMSCs or IPCs twice, on days 1 and 6 . Then splenocytes were isolated from the pre-sensitized mice and co-cultured with the same cells for sensitization with different ratios. No cytolysis difference was observed among groups with different effector/target ratio in either hUCMSC or IPC co-culturing groups ( $\mathrm{P}>0.05)$ (Fig. 3C). When cells co-cultured in the highest effector/target ratio (100:1), the apoptotic rates of hUCMSCs and IPCs were $2.4 \pm 0.44$ and $2.47 \pm 0.66 \%$ respectively, with no difference $(\mathrm{P}>0.05)$ (Fig. 3C). This indicated that hUCMSCs were low immunogenic and immunosuppressive, thus could not activate memory $\mathrm{T}$ cells and cytolysis $\mathrm{T}$ cells in vivo. After the in vitro 
A

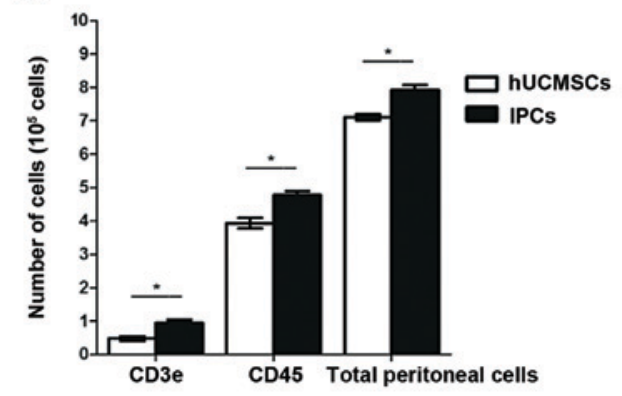

C

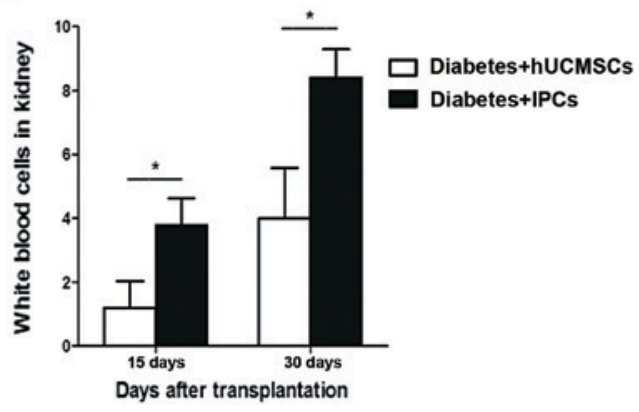

B
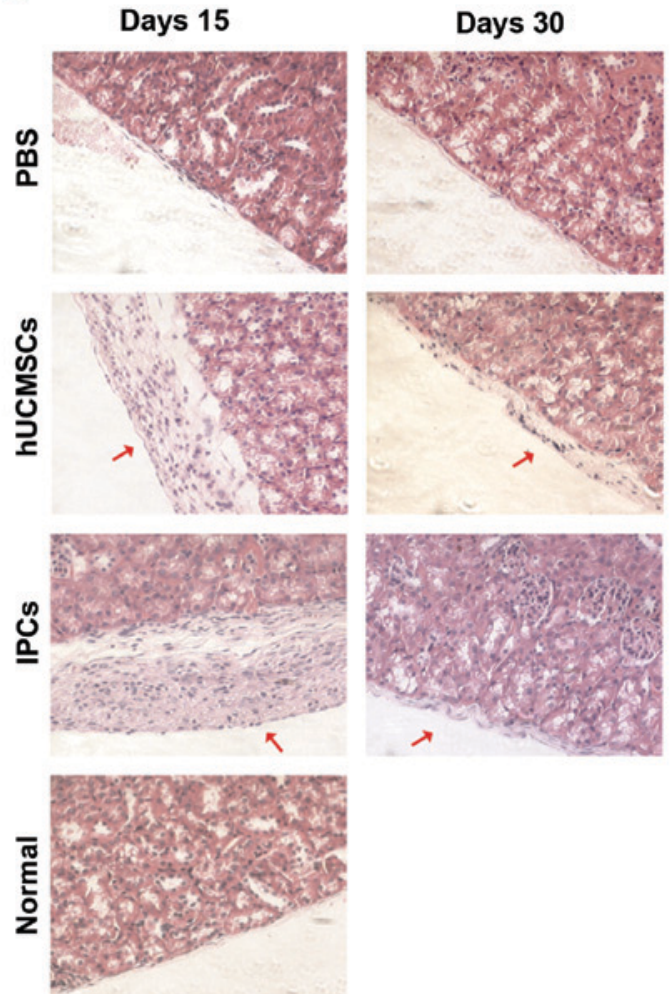

Figure 4. In vivo immunogenicity testing of induced IPCs. (A) Cell population analysis in peritoneal lavage. FACS shows that more CD3e ${ }^{+}$and CD45 ${ }^{+}$cells were detected in the IPC-treated group compared with hUCMSCs treated group. ${ }^{*} \mathrm{P}<0.05$. (B) The pathological H\&E staining of mouse left kidneys after transplantation. The red thick arrows show the hUCMSCs or IPCs, respectively ( $n=4, x 200$ magnification). (C) Representative images of white blood cell counts in the left kidney subcapsular region in 10 high-power fields (x400) per sample at different time-points. * $\mathrm{P}<0.05$. IPCs, insulin-producing cells; hUCMSCs, human umbilical cord mesenchymal stem cells; H\&E, hematoxylin and eosin.

induction, the purified IPCs did not activate immune cells or elicit cytolysis in vivo due to its hypo-immunogenicity.

Cytokine secretion in CML. IFN- $\gamma$, IL-2 and IL-4 are Th1/Th2 cytokines which are very important in mediating and regulating immunity. We tested these cytokines in the supernatants of the co-cultured cells in CML. The results showed that there was no significant difference of cytokine secretion between IPCs and hUCMSC co-culture groups at different ratios (Fig. 3D-F). These results suggested that hUCMSCs and induced IPCs could not activate immune cells and no Th1/Th2 cytokine secretion changes occurred when transplanted the second time.

Immune cells in peritoneal lavage. To determine the acute rejection of hUCMSCs and IPCs, cells were injected into the peritoneal cavity. The peritoneal lavage was extracted and cells positive for leukocyte $\left(\mathrm{CD} 45^{+}\right)$and $\mathrm{T}$ lymphocytes $\left(\mathrm{CD} 3 \mathrm{e}^{+}\right)$ were analyzed by FACS. Total cells in peritoneal lavage extracted from the hUCMSCs group were $7.10 \pm 0.55 \times 10^{5}, 55 \%$ of the cells was $\mathrm{CD}^{2} 5^{+}$, and $6.8 \%$ was $\mathrm{CD} 3 \mathrm{e}^{+}$. Compared to the hUCMSCs group, an increased number of cells were found in peritoneal lavage from the IPCs injection group $(\mathrm{P}<0.05)$, total cells were $7.92 \pm 0.09 \times 10^{5}$, in which $60 \%$ of the cells expressed $\mathrm{CD}^{+} 5^{+}$and $12 \%$ were $\mathrm{CD} 3 \mathrm{e}^{+}$, which was much higher than that in the hUCMSCs group (Fig. 4A) $(\mathrm{P}<0.05)$. This indicates that induced IPCs attract immune cells to infiltrate into the injection sites, showing some immunogenicity in vivo.
Histopathological analysis. On day 15, no leukocyte infiltration was observed in left kidney of hUCMSC-transplanted group, while in the IPCs groups, a small number of immune cells was found in the transplanted region $(\mathrm{P}<0.05)$; on day 30 , in the IPCs group, more immune cells infiltrated to the left kidney compared to day $15(\mathrm{P}<0.05)$, but in the hUCMSCs group, only few immune cells were observed (Fig. 4B and C). This result suggests that the immunogenicity of induced IPCs was enhanced after transplantation in vivo, which could attract leukocyte infiltrate to the transplanted sites.

Reversal of hyperglycemia after IPCs transplantation. After IPCs administration, the BG levels decreased rapidly to $229 \pm 16 \mathrm{mg} / \mathrm{dl}$ by day 12 , and reached $268 \pm 12 \mathrm{mg} / \mathrm{dl}$ by day 30 (Fig. 5A). In the hUCMSC-transplanted group, the BG decreased slightly to $323 \pm 33 \mathrm{mg} / \mathrm{dl}$ by day 12 , and eventually returned to $350 \pm 34 \mathrm{mg} / \mathrm{dl}$, and this ability to reduce BG may be due to tissue repair and paracrine effects. The weight of diabetic mice was improved to some extent in both hUCMSC and IPC treated groups by the end of the study (Fig. 5B). These data showed that the induced IPCs improved the conditions of diabetes.

\section{Discussion}

MSCs can be derived from different tissues, including adipose, bone marrow or umbilical cord (23). MSCs were considered low immunogenic due to their low expression 

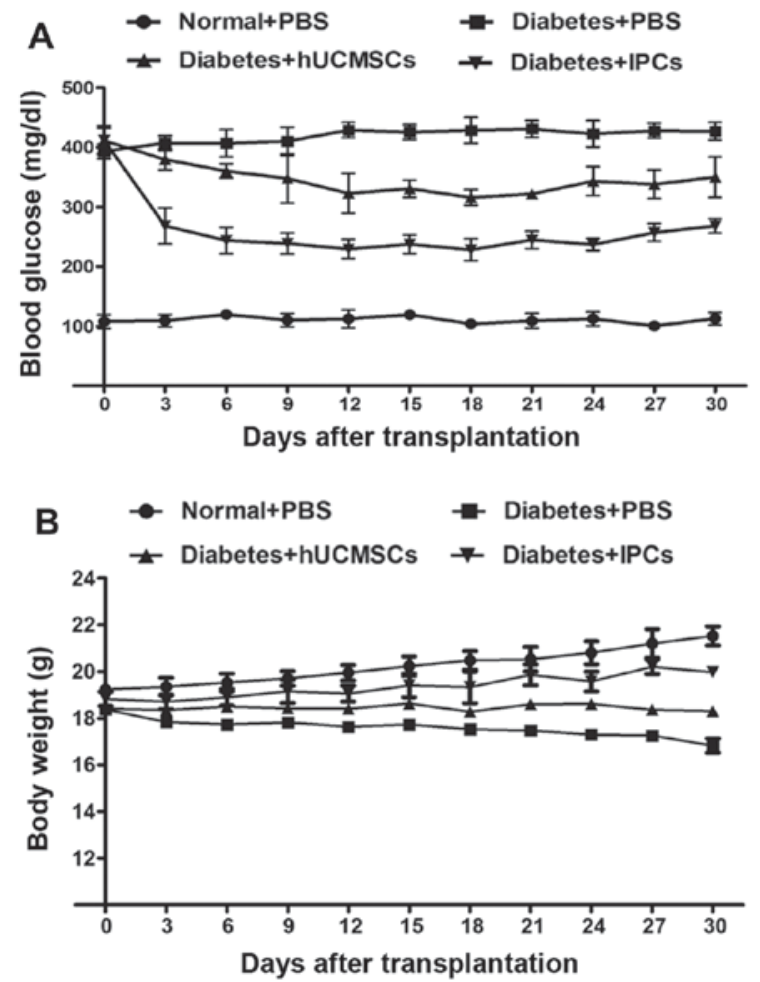

Figure 5. Reversal of hyperglycemia after IPC transplantation. Diabetic mice received hUCMSCs or IPCs infusion under left kidney capsule. (A) The BG levels and (B) weight were assessed every 3 days. IPCs, insulin-producing cells; hUCMSCs, human umbilical cord mesenchymal stem cells.

of immune antigens on the surface $(24,25)$, which makes them hard to be recognized by $\mathrm{T}$ lymphocytes $(6,10)$. In both in vitro and in vivo settings, MSCs can inhibit T-lymphocyte proliferation, suppress proliferation and activation of NK cells and DCs, and reduce the cytokine secretion of T cells and NK cells. They also lower the immune rejections, induce immune tolerance, and thus improve the success rate of organ transplantation (26). However, immunogenicity of MSCs may be enhanced due to the differentiation process and transplantation microenvironments (9-14). After differentiation or transplantation, MSCs may elicit immune responses, thus reducing the survival and further differentiation of the cells, and finally hamper the clinical application of MSCs. In the present study, we successfully induced hUCMSCs to differentiate into functional IPCs by modified induction protocol with the addition of INGAP-pp. Then we investigated the immunological properties of induced IPCs in vitro and immune responses after transplantation into diabetic mice, in order to explore the effects on MSC immunogenicity of induction process and further provide basis for future clinical treatments of diabetes.

Induced IPCs expressed high levels of HLA-ABC, but no HLA-DR and co-stimulatory molecules CD40 and CD80. MHC molecules selectively recognize and bind with antigen peptide, and help presenting antigens. They bind with naïve $\mathrm{T}$ cells and form first signal of T-cell activation. Co-stimulatory molecules CD80 and CD40 produce second signal of T-cell activation. IPCs failed to present antigen to $\mathrm{CD} 4^{+}$cells due to absence of MHC-II on the surface. In one-way, mixed lymphocyte reaction assay antigen-presenting cells (APCs) in allogenic hPBMCs can indirectly present alloantigen to the host lymphocytes, but we did not find allogenic hPBMC proliferation when co-cultured with induced IPCs. This indicated that induced IPCs could not activate naïve $\mathrm{T}$ cells in vitro even with the assistance of exogenous APCs. Therefore, induction of IPCs in vitro did not change the immune phenotype and maintained hypo-immunogenic, which makes them escape the presentation of antigen and fail to activate naïve $\mathrm{T}$ cells. Similarly, hUCMSCs were induced to differentiate into hepatocyte-like cells, similar to its undifferentiated progenitors, it did not express MHC-II molecules and significantly inhibited lymphocyte proliferation (27). MSCs from rabbit bone marrow did not express MHC-II molecules after osteogenesis in vitro, and failed to stimulate allogenic PBMC proliferation and cytolytic T-cell activation, but increased secretion of anti-immunological cytokine IL-10 (28).

In the cell-mediated lysis assay, when hUCMSCs or IPCs were co-cultured with present-sized splenocytes, there was no significant difference of the apoptotic rate between the IPCs and hUCMSCs group. This indicates that IPCs could not activate CTLs and memory $\mathrm{T}$ cells though the expression of MHC-I molecules in the surface of IPCs was higher than that of hUCMSCs. Liu et al (28) transplanted induced osteoblasts from MSCs into rabbits, and observed expression of MHC-II molecule on day 7 post-transplantation, but the transplanted cells survived for 28 days. When fibroblast was transplanted to the same host for the second time, no rejection occurred to the transplanted fibroblasts, indicating no activation of CTLs and memory $\mathrm{T}$ cells (28). Memory $\mathrm{T}$ cell production and activation needs the assistance of $\mathrm{CD} 4^{+} \mathrm{T}$ cells. Studies demonstrated that $\mathrm{CD} 4^{+} \mathrm{T}$ cells did not activate and there was no upregulation of IFN- $\gamma$ and IL-2 secretion after intravenous injection of allogenic MSCs. Our data were in accordance with those results. In our study, compared to undifferentiated hUCMSCs, Th1 cytokines (IL- 2 and IFN- $\gamma$ ) and Th2 cytokines (IL-4) did not change in differentiated IPCs co-culture group, indicating that the balance between Th1 and Th2 was not broken by induced IPCs and the activation of T cells (29).

Differentiated IPCs exhibit low immunogenicity in vitro, but how they behave after transplantation to the host remains to be determined. Additionally, whether they elicit inflammatory responses and cause acute or chronic rejection should be investigated. In the present study, we found leukocyte infiltration to the peritoneal lavage and a slight upregulation of $\mathrm{T}$ lymphocytes $4 \mathrm{~h}$ post-IPC injection to the peritoneal cavity. This may be due to the upregulation of MHC-I in differentiated IPCs, leading to the activation of the monocyte-macrophage system (innate immunity) and the infiltration of immune cells. Similarly, histopathological analysis revealed that a few IPCs were found to be alive on day 14 after transplantation under the capsule of the left kidney. Leukocyte infiltration to the transplanted sites was observed in IPCs but hUCMSCs transplanted mice. On day 30, very few IPCs survived but elevated inflammatory cells infiltration was seen in the transplanted region. On the contrary, almost no immune cell infiltration was found in hUCMSCs group. The above demonstrated that IPCs became immunogenic after transplantation, which might be the consequences of the disease microenvironment. Accordingly, BG reduced rapidly after IPCs transplantation 
into diabetic mice, but eventually returned to hyperglycemia again due to the loss of transplanted IPCs. The possible explanation may be the enhancement of IPC immunogenicity by the disease microenvironment.

Our previous data demonstrated that allogenic bone marrow-derived MSCs continued to differentiate into IPCs after transplantation under pancreatic capsule of diabetic rats. However, with the occurrence of differentiation, MHC-II expression was positive on MSCs and alloantibodies were detected in the serum, indicating that differentiation initiates an immune 'switch' that alters the immune characteristics of MSCs in vivo (30). Huang et al found that MSCs started to express myocardiocyte-specific markers and also the MHC-I and MHC-II after transplanted to infracted myocardium (12). Microenvironment in the disease recipients could promote differentiation of MSCs, and change its immunopreviliged state in vivo (12).

It has been demonstrated that the body was in an inflammatory state in the STZ-induced diabetes model. The secretion of inflammatory cytokines possibly induced immune antigens expression in the surface of IPCs, thus eliciting immune rejection. IFN- $\gamma$ has been demonstrated to induce MHC-I and MHC-II expression on the surface of MSCs in vitro and in vivo (31-33). To clarify the underlying mechanism of immunological changes after IPC differentiation, the relationship between disease immune microenvironment and immune characteristic alteration should be examined.

In conclusion, although the immunogenicity of IPCs derived from hUCMSCs was not altered in vitro, they can become immunogenic by the interaction with disease microenvironment and induce inflammatory responses and recruit immune cell infiltration into the transplanted sites.

\section{Acknowledgements}

This study was supported by the National 973 Special Plan of China (no. 2007CB516811), the National Natural Science Foundation of China (no. 30772042), the Natural Science Foundation of Guangdong (no. 6027540) and the Science and Technology Profect of Shenzhen (no. 201001005).

\section{References}

1. Shapiro AMJ, Lakey JRT, Ryan EA, Korbutt GS, Toth E, Warnock GL, Kneteman NM and Rajotte RV: Islet transplantation in seven patients with type 1 diabetes mellitus using a glucocorticoid-free immunosuppressive regimen. N Engl J Med 343: 230-238, 2000.

2. Saidi RF: Current status of pancreas and islet cell transplantation. Int J Organ Transplant Med 3: 54-60, 2012.

3. Xie H, Wang Y,Zhang H, Qi H,Zhou H and Li FR: Role of injured pancreatic extract promotes bone marrow-derived mesenchymal stem cells efficiently differentiate into insulin-producing cells. PLoS One 8: e76056, 2013.

4. Prabakar KR, Domínguez-Bendala J, Molano RD, Pileggi A, Villate S, Ricordi C and Inverardi L: Generation of glucoseresponsive, insulin-producing cells from human umbilical cord blood-derived mesenchymal stem cells. Cell Transplant 21: 1321-1339, 2012

5. Gabr MM, Zakaria MM, Refaie AF, Ismail AM, Abou-ElMahasen MA, Ashamallah SA, Khater SM, El-Halawani SM, Ibrahim RY, Uin GS, et al: Insulin-producing cells from adult human bone marrow mesenchymal stem cells control streptozotocin-induced diabetes in nude mice. Cell Transplant 22: 133-145, 2013.
6. Uccelli A, Moretta L and Pistoia V: Mesenchymal stem cells in health and disease. Nat Rev Immunol 8: 726-736, 2008.

7. Poncelet AJ, Vercruysse J, Saliez A and Gianello P: Although pig allogeneic mesenchymal stem cells are not immunogenic in vitro, intracardiac injection elicits an immune response in vivo. Transplantation 83: 783-790, 2007.

8. English K, French A and Wood KJ: Mesenchymal stromal cells: facilitators of successful transplantation? Cell Stem Cell 7: 431-442, 2010.

9. Lohan P, Coleman CM, Murphy JM, Griffin MD, Ritter T and Ryan AE: Changes in immunological profile of allogeneic mesenchymal stem cells after differentiation: should we be concerned? Stem Cell Res Ther 5: 99, 2014.

10. Le Blanc K, Tammik C, Rosendahl K, Zetterberg E and Ringdén O: HLA expression and immunologic properties of differentiated and undifferentiated mesenchymal stem cells. Exp Hematol 31: 890-896, 2003.

11. Liu CT, Yang YJ, Yin F, Wang X, Yu XH, Wang QH, Wang XL and Xie M: The immunobiological development of human bone marrow mesenchymal stem cells in the course of neuronal differentiation. Cell Immunol 244: 19-32, 2006.

12. Huang XP, Sun Z, Miyagi Y, McDonald Kinkaid H, Zhang L, Weisel RD and Li RK: Differentiation of allogeneic mesenchymal stem cells induces immunogenicity and limits their long-term benefits for myocardial repair. Circulation 122: 2419-2429, 2010.

13. Technau A, Froelich K, Hagen R and Kleinsasser N: Adipose tissue-derived stem cells show both immunogenic and immunosuppressive properties after chondrogenic differentiation. Cytotherapy 13: 310-317, 2011.

14. Chen X, McClurg A, Zhou GQ, McCaigue M, Armstrong MA and Li G: Chondrogenic differentiation alters the immunosuppressive property of bone marrow-derived mesenchymal stem cells, and the effect is partially due to the upregulated expression of B7 molecules. Stem Cells 25: 364-370, 2007.

15. Dhingra S, Li P, Huang XP, Guo J, Wu J, Mihic A, Li SH, Zang WF, Shen D, Weisel RD, et al: Preserving prostaglandin E2 level prevents rejection of implanted allogeneic mesenchymal stem cells and restores postinfarction ventricular function. Circulation 128 (Suppl 1): S69-S78, 2013.

16. Seshareddy K, Troyer D and Weiss ML: Method to isolate mesenchymal-like cells from Wharton's Jelly of umbilical cord. Methods Cell Biol 86: 101-119, 2008.

17. Wang HS, Shyu JF, Shen WS, Hsu HC, Chi TC, Chen CP, Huang SW, Shyr YM, Tang KT and Chen TH: Transplantation of insulin-producing cells derived from umbilical cord stromal mesenchymal stem cells to treat NOD mice. Cell Transplant 20: 455-466, 2011.

18. Li J, Wang Y, Yu X, Chen H, Wu Y, Han X, Guo X, Zhang C, Chen Q, Chen J, et al: Islet neogenesis-associated protein-related pentadecapeptide enhances the differentiation of islet-like clusters from human pancreatic duct cells. Peptides 30: 2242-2249, 2009.

19. Pfaffl MW: A new mathematical model for relative quantification in real-time RT-PCR. Nucleic Acids Res 29: e45, 2001.

20. Morandi F, Levreri I, Bocca P, Galleni B, Raffaghello L, Ferrone S, Prigione I and Pistoia V: Human neuroblastoma cells trigger an immunosuppressive program in monocytes by stimulating soluble HLA-G release. Cancer Res 67: 6433-6441, 2007.

21. Lukowiak B, Vandewalle B, Riachy R, Kerr-Conte J, Gmyr V, Belaich S, Lefebvre J and Pattou F: Identification and purification of functional human beta-cells by a new specific zinc-fluorescent probe. J Histochem Cytochem 49: 519-528, 2001.

22. Ye J, Liao YT, Jian YQ, Zhang XD, Wei P, Qi H, Deng CY and Li FR: Alpha-1-antitrypsin for the improvement of autoimmunity and allograft rejection in beta cell transplantation. Immunol Lett 150: 61-68, 2013

23. Wang HW, Lin LM, He HY, You F, Li WZ, Huang TH, Ma GX and Ma L: Human umbilical cord mesenchymal stem cells derived from Wharton's jelly differentiate into insulin-producing cells in vitro. Chin Med J (Engl) 124: 1534-1539, 2011.

24. Casado JG, Gomez-Mauricio G, Alvarez V, Mijares J, Tarazona R, Bernad A and Sanchez-Margallo FM: Comparative phenotypic and molecular characterization of porcine mesenchymal stem cells from different sources for translational studies in a large animal model. Vet Immunol Immunopathol 147: 104-112, 2012.

25. Lee JM, Jung J, Lee HJ, Jeong SJ, Cho KJ, Hwang SG and Kim GJ: Comparison of immunomodulatory effects of placenta mesenchymal stem cells with bone marrow and adipose mesenchymal stem cells. Int Immunopharmacol 13: 219-224, 2012. 
26. Shi Y, Hu G, Su J, Li W, Chen Q, Shou P, Xu C, Chen X, Huang Y, Zhu Z, et al: Mesenchymal stem cells: A new strategy for immunosuppression and tissue repair. Cell Res 20: 510-518, 2010.

27. Zhao Q, Ren H, Li X, Chen Z, Zhang X, Gong W, Liu Y, Pang T and Han ZC: Differentiation of human umbilical cord mesenchymal stromal cells into low immunogenic hepatocyte-like cells. Cytotherapy 11: 414-426, 2009.

28. Liu H, Kemeny DM, Heng BC, Ouyang HW, Melendez AJ and Cao T: The immunogenicity and immunomodulatory function of osteogenic cells differentiated from mesenchymal stem cells. J Immunol 176: 2864-2871, 2006.

29. Amirzargar A, Lessanpezeshki M, Fathi A, Amirzargar M, Khosravi F, Ansaripour B and Nikbin B: TH1/TH2 cytokine analysis in Iranian renal transplant recipients. Transplant Proc 37: 2985-2987, 2005.

30. Sadeghi M, Daniel V, Naujokat C, Schmidt J, Mehrabi A, Zeier M and Opelz G: Evidence for IFN- $\gamma$ up- and IL-4 downregulation late post-transplant in patients with good kidney graft outcome. Clin Transplant 21: 449-459, 2007.
31. Stubbendorff M,Deuse T,Hua X,Phan TT,Bieback K, Atkinson K, Eiermann TH, Velden J, Schröder C, Reichenspurner H, et al: Immunological properties of extraembryonic human mesenchymal stromal cells derived from gestational tissue. Stem Cells Dev 22: 2619-2629, 2013.

32. Zhang X, Tang T, Shi Q, Fernandes JC and Dai K: The immunologic properties of undifferentiated and osteogenic differentiated mouse mesenchymal stem cells and its potential application in bone regeneration. Immunobiology 214: 179-186, 2009.

33. Elias D, Prigozin H, Polak N, Rapoport M, Lohse AW and Cohen IR: Autoimmune diabetes induced by the beta-cell toxin STZ. Immunity to the $60-\mathrm{kDa}$ heat shock protein and to insulin. Diabetes 43: 992-998, 1994. 